\title{
POLARIZATION MEASUREMENTS IN B5, L1 34 AND HEILES CLOUD 2 AND EVIDENCE OF NEWLY BORN STARS
}

\author{
U.C. Joshi, P.V.Kulkarni, M.R. Deshpande, A. Sen and \\ A.K. Kulshrestha \\ Physical Research Laboratory \\ N avr angpura \\ A hmedabad- 380009 \\ India
}

Polarization measurement of the background stars in the region of dark globules is important to study the magnetic field geometry and grain's characteristics in the globule. These parameters are important for the formation and evolution of dark globules. We made polarimetric observations of stars in three nearby dark clouds B5, L134 and Heiles Cloud 2. Polarization measurements of stars in the region of B5 were made with 'MINIPOL' (Frecker and Serkowski, 1976) on 61" tel escope of University of Arizona. Observations for the stars in the region of L134 and Heiles Cloud 2 were made using PRL polarimeter (Deshpande et al. 1985) on 1 meter telescope of Indian Institute of Astrophysics, Bangalore. Results are presented and discussed here. Figure 1 shows the polarization vectors projected on the sky plane for the above globules.

We assume Davis-Greenstein mechanism for grain alignment. In this situation polarization vectors are the direction of projected magnetic field. In B5 polarization vectors are generally aligned in the $\mathrm{NE}$ and $\mathrm{SW}$ direction, except for the $\mathrm{NW}$ region where the vectors are more or less perpendicular to the polarization vectors in other region. Detailed discussion on B5 is given elsewhere (Joshi et al. 1984). Following are the main inferences -

a) Strong st ellar wind from embeded IR sources (Beichnann et al. 1984) especially from IRS1 has retarded the collapse along NESW direction, which is also the direction of rotation axis.

b) the central object (IRS1?) form with a torus around it; the orientation of torus being perpendicular to the rotation axis. The torus collimates the mass loss from the central object, which has further restricted the collapse. The magnetic field is constricted by the collapse and follows the torus. The polarization vector for stars 13,16 and 17 may be thus explained. 
c) Some stars (e.g. star no. 6 and 16) show $\lambda$-dependence in position angle. This is possible when light passes through inhomogeneous regions, with various orientation of dust grains (Coyne, 1974). The anisotropic stellar wind can produce inhomogeneity ${ }_{h}^{1}$ the nearby regions of embeded IR sources. The mass loss from newly born stars have perhaps disturbed the otherwise homogeneous distribution of grain s significantly.

Polarization vectors in dark clouds L1 34 and Heiles Cloud 2 show high degree of alignment. This probably indicates either the star formation has not yet set in or has not reached to a stage where vigorous mass loss is expected. Degree of polarization in Heiles Cloud 2 is quite high $(P \sim 6 \%)$ which means the degree of alignment is also high. This is possible only if the magnetic field in the surrounding region is high or the disturbing factors are at minimum.

\section{References}

Beichmann, C.A., Jennings, R.E., Emerson, J.P., Baud, B., Harris, S., Rowan-Robinson, M., Aumann, H. H., Gautier, T.N., Gillet, F.C., and Young, E. : 1984, A strophys. J. 278, L45 Coyne, G.V. : 1976, A stron. J. 79, 565

Deshpande, M. R., Jo shi, U.C., Kulshrestha, A.K., Banshidhar., Vadhe r, N. M. , Mazumdar, H. So, Pradhan, S. N., and Shah, C. R.: 1985, Bull. Astron. Soc. India 13, 157

Frecker, J.E. and Serkowski, K. : $\overline{1976}$, Appl. Opts., 15, 605 Joshi, U.C., Kulkarni, P.V., Bhatt, H.C., Kulshrestha, A.K. and

Des hpande, M。 R。 : 1985, Mon. Not. R. A stron. Soc. 215, 275 

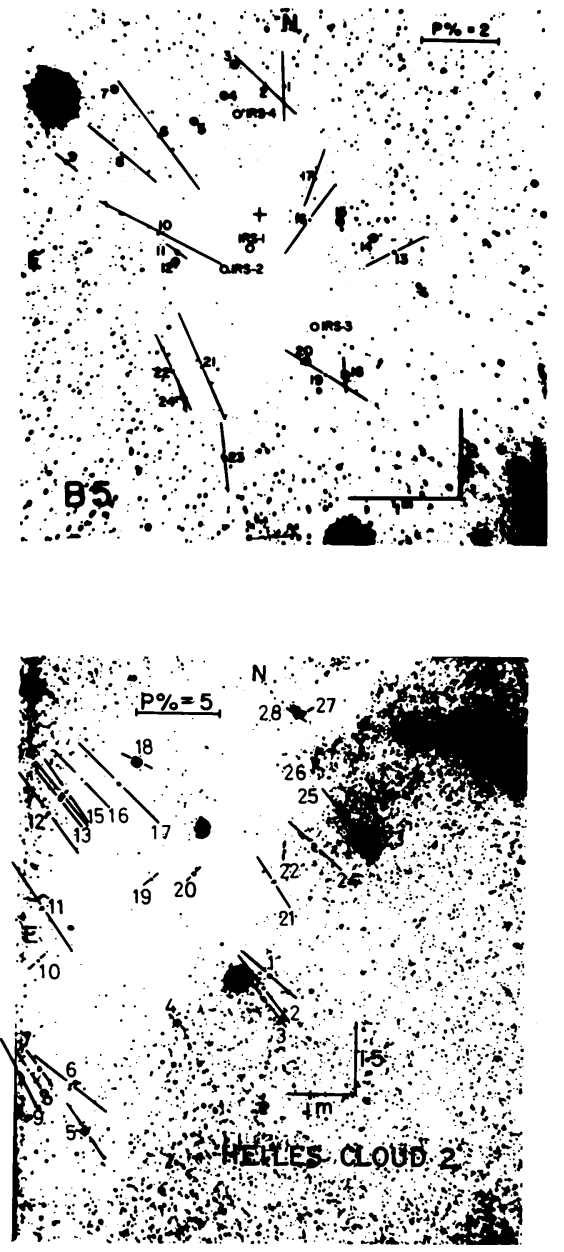

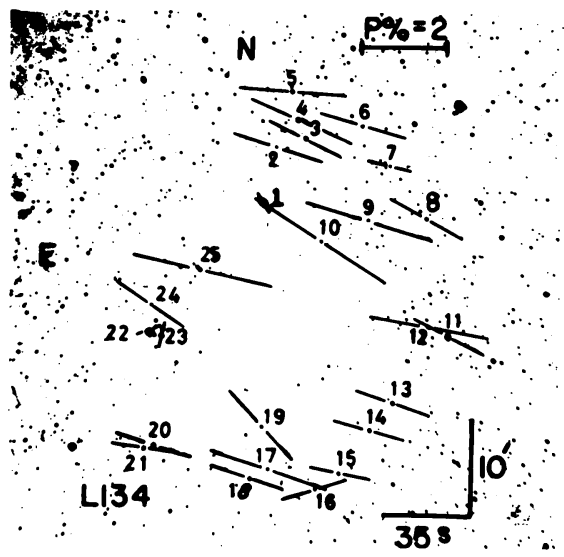

Figure 1. Polarization map for globules B5, L1 34 and Heiles cloud 2. Polarization vectors are superimposed on the star fields, taken from Palomar Sky Survey p rints. 